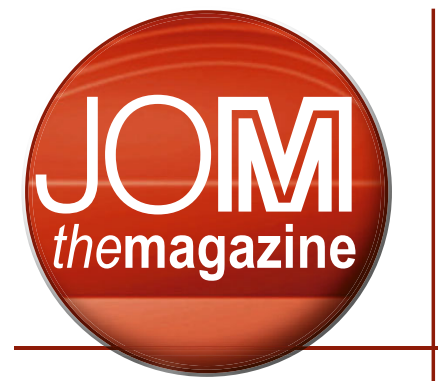

\section{member news}
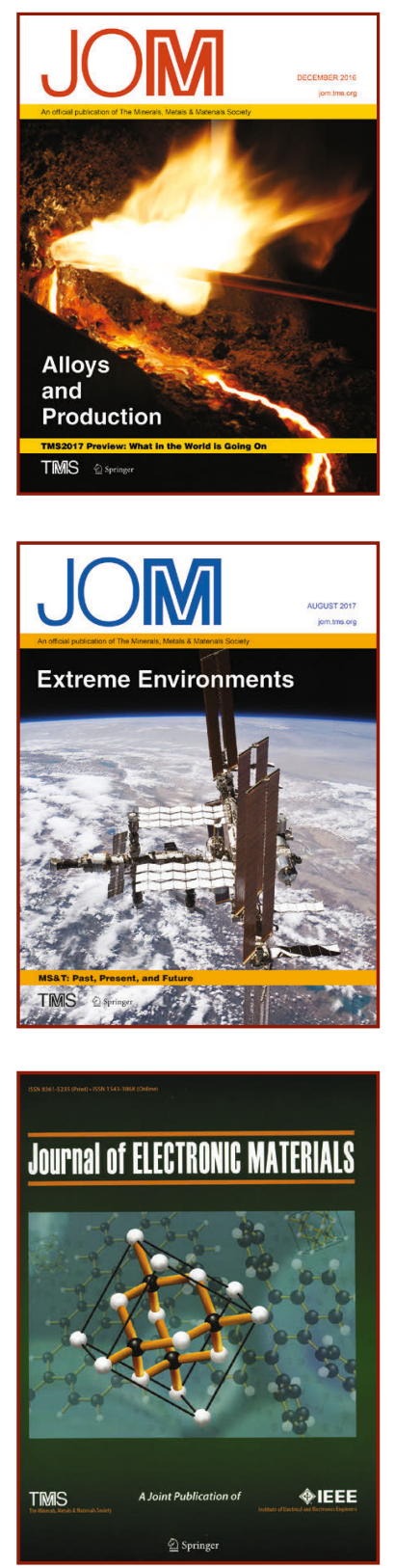

250

\title{
2018 JOM and JEM Best Paper Awards; TMS Members Inducted into NAI; TMS Welcomes New Members
}

\section{TMS Divisions Announce 2018 JOM, JEM Best Paper Award Recipients}

The TMS Light Metals Division (LMD) and Structural Materials Division (SMD) will present their 2018 JOM Best Paper Awards during their respective division luncheons at the TMS 2018 Annual Meeting \& Exhibition (TMS2018), March 11-March 15, in Phoenix, Arizona. The JOM Best Paper Awards recognize the excellence of papers published in the preceding year's volume of $J O M$ that exemplify the application of science in solving a practical problem in either a light metals or structural materials related technical topic.

The recipients of the 2018 LMD JOM Best Paper Award are Alexei Vinogradov, Norwegian University of Science \& Technology, Trondheim, Norway, and Kristian Máthis, Charles University, Prague, Czech Republic, for "Acoustic Emission as a Tool for Exploring Deformation Mechanisms in Magnesium and Its Alloys In Situ" published in the December 2016 JOM. In the article, the authors review the capacity of the advanced acoustic emission (AE) technique to understand the interplay between two primary deformation mechanisms - dislocation slip and twinning - in real time scale.

The 2018 SMD JOM Best Paper Award goes to Sudhanshu S. Singh, Indian Institute of Technology Kanpur, India, Tyler J. Stannard and Nikhilesh Chawla, both from Arizona State University, Tempe, Arizona, USA, and Xianghui Xiao,
Argonne National Laboratory, Lemont, Illinois, USA. Their August 2017 paper, "In Situ X-ray Microtomography of Stress Corrosion Cracking and Corrosion Fatigue in Aluminum Alloys," presented recent 4D studies on stress corrosion cracking and corrosion-fatigue behavior of Al7075 alloys using x-ray synchrotron tomography.

The TMS Functional Materials Division (FMD) will also recognize the 2018 JEM Best Paper Award recipients for outstanding contributions to the Journal of Electronic Materials (JEM) at the FMD Council meeting held during TMS2018. Si Chen, Tong An, Fei Qin, Beijing, and Pei Chen, all from Beijing University of Technology, China, received the award for "Microstructure Evolution and Protrusion of Electroplated Cu-Filled ThroughSilicon Vias Subjected to Thermal Cyclic Loading", published in the October 17 issue of JEM. In this work, thermal cycling tests were carried out to identify how microstructure affects protrusion during thermal cycling.

The 2018 JOM and JEM Best Paper articles are available for free download to TMS members. Log in to www.tms.org/journals to gain free electronic access to $J O M$ and $J E M$, as well as all other TMS journals, including Metallurgical and Materials Transactions $A$ and $B$, Integrating Materials and Manufacturing Innovation, and Journal of Sustainable Metallurgy.

Share the good news about your professional accomplishments! Contact Lynne Robinson, JOM Magazine Editor, at Irobinson@tms.org. Please note that only news submitted by current TMS members will be considered. 


\section{TMS Members Inducted into the National Academy of} Inventors

TMS congratulates the following members for being inducted as Fellows into the National Academy of Inventors (NAI). They will be formally recognized at a ceremony slated for April 5, 2018 in Washington, D.C.

Diran Apelian is the AlcoaHowmet Professor of Mechanical Engineering at Worcester Polytechnic Institute (WPI) and the founding director of WPI's Metal Processing Institute. He was the 2008 TMS President and a 2006 TMS Fellow, and has received the TMS Bruce Chalmers Award and delivered the 2004 TMS/ASM Distinguished Lecture in Materials and Society, among his many awards and honors.

Zhigang Z. Fang is a professor at the University of Utah. A TMS member since 1992, he has authored/co-authored more than 120 publications and holds more than 30 U.S. patents.

Mark C. Hersam is the Walter P. Murphy Professor of Materials Science and Engineering at Northwestern University and Director of the Northwestern University Materials Research Center. He is the recipient of the 2006 AIME Robert Lansing Hardy Award and a 2014 recipient of a MacArthur Fellowship.

M. Parans Paranthaman is a Corporate Fellow and a Group Leader of Materials

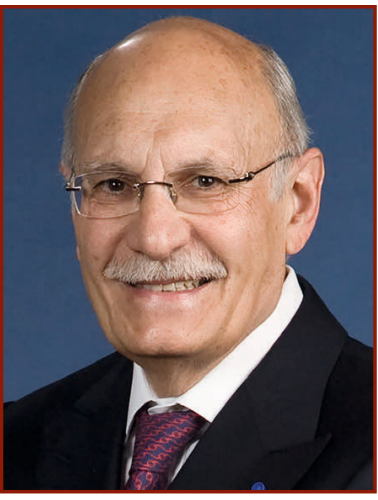

Diran Apelian

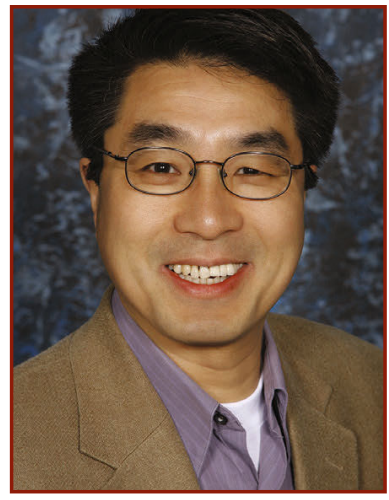

Zhigang Z. Fang

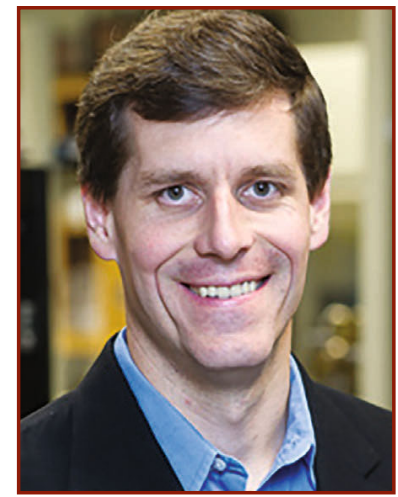

Mark C. Hersam
Chemistry at the Chemical Sciences Division at the Oak Ridge National Laboratory (ORNL), and has been an active TMS member since 1993.

Richard W. Siegel is the Robert W. Hunt Professor of Materials Science and Engineering at the Rensselaer Polytechnic Institute. An active TMS member since 1992, he has authored or coauthored more than 300 articles and patents, edited ten books, and presented more than 500 invited lectures around the world.

The goal of NAI is to recognize inventors who have created patents that enhance the visibility of academic technology and innovation, encourage the disclosure of intellectual property, and educate and mentor innovative students. The rank of Fellow is the highest honor that NAI confers.

\section{TMS Welcomes New Members}

The TMS Board of Directors approved professional membership for the following individuals at its December 2017 meeting. Please join us in congratulating and welcoming them to all the privileges and benefits of TMS membership.

Ahmad, Iftikhar; Lambada Technologies, United States

Alsahli, Abdulmohsen; Saudi Basic Industries Corp. (Sabic), Saudi Arabia

Anderson, Kenneth R.; Naval Nuclear Laboratory, United States

Arthur, Nana K.; Council for Scientific and Industrial Research, South Africa

Arvisais, Jean-Paul; Aluminerie Alouette Inc., Canada
Azadfar, Mohammadali; Washington State University, United States

Baake, Egbert; Leibniz University of Hannover, Germany

Bakas, Michael P.; Army Research Office, United States

Balogun, Yunusa A.; NSWC Crane Division, United States

Bamiduro, Olu; Solar Way Solutions, United States

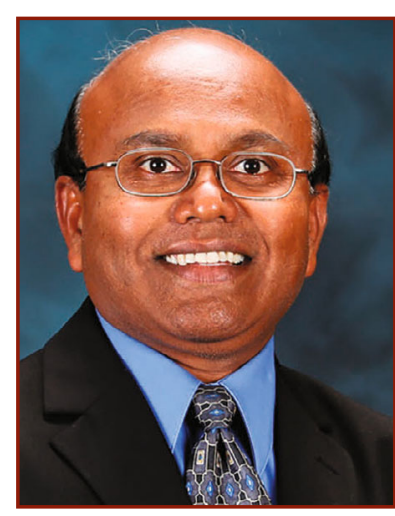

M. Parans Paranthaman

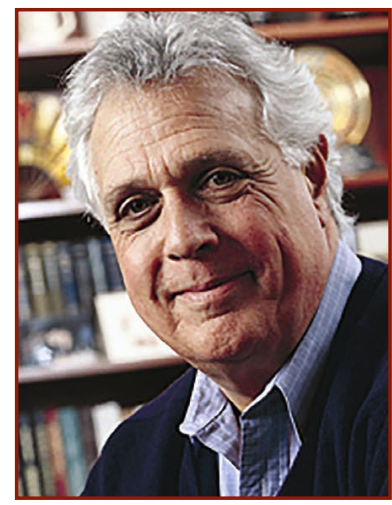

Richard W. Siegel 
Beaman, Joseph J.; University of Texas at Austin, United States

Becerra, Ana Maria; Hatch, Canada

Bellot, Jean-Pierre; Lorraine University, France

Bermingham, Michael; The University of Queensland, Australia

Berube, Jean-Pierre; Aluminerie Alouette Inc., Canada

Bianculli, Steven R.; United States Steel, United States

Bing, Ni; Central Iron and Steel Research Institute, China

Bishop-Moser, Joshua; Mforesight, United States

Bjorkman, Bo M.; Lulea University of Technology, Sweden

Bolduc, Emile; Rio Tinto - Jonquiere Works, Canada

Bouhabila, El Hani; Solios Environment SA, France

Bourdon, Annie; Aluminerie Alouette Inc., Canada

Braeuhaus, Dennis; Heraeus Quarzglas, Germany

Brisson, Pierre-Yves, Rio Tinto Aluminium, Canada

Bristow, James; NOVA Geotechnical \& Inspection Services PLLC, United States

Brush, Lucien N.; University of Washington, United States

Bryan, David; ATI Specialty Material, United States

Burns, Madison; Baker Hughes, Germany

Campbell, Geoffrey H.; Lawerence Livermore National Laboratory, United States

Camperlingo, Flavio Eduardo; Petrocoque S/A, Brazil

Caspers, Christopher; Federal-Mogul, United States

Chalmers, lan; Alkane Resources Ltd, Australia

Chen, Aiping; Los Alamos National Laboratory, United States
Chen, Wei; Northwestern University, United States

Chowdhri, Abhinav; Supreme Special Steels, India

Christman, Brett A.; Naval Nuclear Laboratory, United States

Cibula, Matthew A.; Ampere Scientific, United States

Couvrat, Mathieu; Manoir Pitres, France

Cuevas, Fermin; ICMPE - CNRS, France

Dai, Chen; VJ Technologies, United States

D’Amore, Lisa L.; BMPC/Knolls Atomic Power Laboratory, United States

De Neufville, John; Eutectix, United States

Debruin, Mark E.; Skuld LLC, United States

Dechent, Matthias; Trimet Aluminium SE, Germany

Deng, Xin; University of Science \& Technology Liaoning, China

Depond, Philip; Lawrence Livermore National Laboratory, United States

Dolok Saribu, Wallter Z.; PT Indonesia Asahan Alum (PERSERO), Indonesia

Dong, Yanwu; Northeastern University, China

Drake, Michael; PCC Structurals, United States

Dubelman, Meredith; GE Additive, United States

Duvernay, Amélie; Five Solios, France

Eggert, Roderick; Colorado School of Mines, United States

Eickoff, Moritz; RWTH Aachen University, Germany

Ekiert, Thomas; Riverside Research, United States

Engler-Pinto, Carlos C.; Ford Motor Company, United States

Enright, Phil; N-Tec Limited, United Kingdom

Evans, David G.; Special Metals Corp., United States

Everett, David D.; Strohecker Inc., United States
Faltens, Tanya; Nanohub/Purdue University, United States

Ferguson, Gehn; U.S. Army Research Laboratory, United States

Ferrer, Laurent; SAFRAN, France

Fezi, Kyle; Fort Wayne Metals, United States

Fu, Engang; Peking University, China

Gao, Jianrong; Northeastern University, China

Gavras, Sarkis; Magic, HelmholtzZentrum Geesthacht, Germany

Gazanion, Fabienne; Rio Tinto, Canada

Geng, Xin; Northeastern University, China

Gingras, Catherine; Rio Tinto, Canada

Gockel, Brian; U.S. Air Force, United States

Graham, Joseph; Missouri University of Science and Technology, United States

Griffith, David; Global Advanced Metals, United States

Gruber, Jason A.; Naval Nuclear Laboratory, United States

Gu, Yejun; Johns Hopkins University, United States

Guidoin, Robert; Laval University, Canada

Guo, Juchen; University of California Riverside, United States

Haghshenas, Meysam; University of North Dakota, United States

Hans, Stephane; Aubert \& Duval, France

Hansén, Thorbjörn; Swerea MEFOS $A B$, Sweden

Harris, Karen E.; Vitro Architectural Glass, United States

Hartmann, Thomas; Vacuumschmelze, Germany

Hassani, Mostafa; Massachusetts Institute of Technology, United States

Hatch, Gareth; Innovation Metals Corp., Canada 
He, Fei; Avery Dennison, United States

$\mathrm{He}$, Zhijun; University of Science and Technology Liaoning, China

Hemeryck, Romain; Five Solios, France

Hensgen, Lars; Tribotecc GmbH, Austria

Horn, Tim; North Carolina State University, United States

Hoseinian Yousef, Taymaz; Norsk Hydro ASA, Norway

Hulbert, Leland; BMPC, United States

Humphreys, Colin; University of Cambridge, United Kingdom

Jagdale, Vijay; United Technologies Research Center, United States

Jain, Vaibhav; Defense Logistics Agency, United States

Jardy, Alain; CNRS/LabEx DAMAS, France

Jiang, Zhouhua; Northeastern University, China

Kaku(Hao), Kyo(Jing); MC Zhenjiang Anode Solutions Co., Ltd, China

Karimi Sibaki, Ebrahim; University of Leoben, Austria, Austria

Kashar, Lawrence; Kashar Technical Services, Inc., United States

Ke, Yucheng; China

Keles, Ozgur; San Jose State University, United States

Kelley, Douglas; University of Rochester, United States

Kennedy, James C.; Three Consulting, United States

Kharicha, Abdellah; Montauniversität, Austria

Kim, Dae Hyun; SeAH, South Korea

Kim, Jinkyung; Postech, South Korea

King, Paul E.; Ampere Scientific, United States

Kish, Joseph; McMaster University, Canada

Koch, Ralf; Vacuumschmelze GmbH \& Co. KG, Germany

Kornecki, Michael; U.S. Army

Research Laboratory, United States
Kraly, Andreas; Lkr Leichtmetallkompetenzzentrum Ranshofen, Austria

Kruz, Andrew; U.S. Army, United States

Kubin, Michael; INTECO Melting and Casting, Austria

Kui, Wang; MC Zhenjiang Anode Solutions Co., China

Kurose, Ryo; Daido Steel America Inc., United States

Kwon, Soon II; Republic of Korea, South Korea

Laflamme, François; Aluminerie Alouette Inc., Canada

Lafrance, Jacques; Aluminerie Alouette Inc., Canada

Lagace, Charles; Aluminerie Alouette Inc., Canada

Lauzon, Julien; Alcoa, Canada

Lavergne, Francis-Joe, Alcoa, Canada

Lejeune, Zorabel M.; Oxbow Calcining LLC, United States

Leon, Marco F.; Universidad San Fancisco De Quito, Ecuador

Leonard, Guillaume; Rio Tinto Alcan ARDC, Canada

Leonard, Eric L.; Elemental Metals, United States

Leon-Brito, Neliza; Los Alamos National Laboratory, United States

Leskovec, Simon; SIJ Metal Ravne, Slovenia

Levey, Christophe G.; Thayer Engineering at Dartmouth, United States

Levkov, Leonid; JSC "RPA CNITMASH," Russian Federation

Li, Jing; University of Science \& Technology Beijing, China

Li, Juntao; China Iron \& Steel Research, China

Li, Maosheng; IAPCM, China

Li, Wanming; Northeastern University, China

Li, Wenxian; Shanghai University, China

Li, Yang; Northeastern University, China

Lim, Mary Lyn; PPG, United States
Lippard, Henry E.; ATI Allvac, United States

Lister, Michael; Flowserve, United States

Litzelman, Scott J.; United States

Liu, Fubin; Northeastern University, China

Liu, Zhonggiu; Northeastern University, China

Low, Rachael, Solar Turbines Inc.; United States

Lu, Haiyan; Institute of Materials China, China

Machaka, Ronald; Council for Scientific and Industrial Research, South Africa

Mahmoud, Morsi Mohamed; King Fahd University of Petroleum, Saudi Arabia

Maiorov, Boris; Los Alamos National Laboratory, United States

McCarthy, Ben; Morgan Advanced Materials, United Kingdom

McCurley, Peyton C.; Oxbow Calcining LLC, United States

McDonald, Neill; MetaFensch, France

Mckinnell, Jim; HP Inc., United States

Mcmillen, Mark, PPG Industries, United States

Merriman, Colin C.; Washington State University, United States

Michelhaugh, Richard T.; Department of Energy, United States

Moll, Amy J.; Boise State University, United States

Morissette, Francois; Rio Tinto, Canada

Motoyasu, Genjiro; Chiba Institute of Technology, Japan

Moyer, Bruce; Oak Ridge National Laboratory, United States

Moyer, Laura E.; Lehigh University, United States

Mubarok, Arif; PPG, United States

Nair, Bindu G.; University of Birmingham, United Kingdom

Nasir, Tanzim; Acuren Group Inc., Canada 
Nulwala, Hunaid; Liquid Ion Solutions, United States

Oh, Sanghun; SeAh Changwon Integrated Special Steel, South Korea

Oppedal, Andrew L.; Mississippi State University, United States

Otminski, Jordan; Ledvance, United States

Ottesen, Petur; Nordural, Iceland

Pappula, Laxminaray; Osmania University, India

Pavlina, Erik J.; AK Steel Corporation, United States

Pintowantor, Sungging; Institut Teknologi Sepuluh, Indonesia

Powell, Shane; Universal Stainless, United States

Raghunathan, Narsimhan; Touchstone Testing Lab LLC, United States

Rainville, Kathia; Rio Tinto, Canada

Rajagopalan, Sudhir; Siemens Power Generation, United States

Raju, Selva; Army Research Laboratory, United States

Raub, Joseph N.; Century Aluminum, United States

Reynolds, William T.; Virginia Polytechnic Institute and State University, United States

Rock, Christophe D.; Center of Additive Manufacturing \& Logistics, United States

Rodek, Eugene; SPI Supplies, United States

Roudant, Julien; ECM USA, United States

Roy, Rose; Xtalic Corporation, United States

Ruppert, Jean; Safran, France

Salesse, Julie; Aluminerie Alouette Inc., Canada

Samant, Rutuja; EWI, United States

Sands, Thomas M.; Carpenter Technology Corporation, United States

Sansone, Andrea; Lucideon, United Kingdom
Sato, Takashi; Japan Casting and Forging, Japan

Saylor, David A.; USFDA, United States

Scheriau, Alexander; INTECO Melting and Casting, Austria

Schneider, Reinhold; FH Forschungs und Entwicklungs, Austria

Shaarawi, Mohamme; Hewlett Packard, United States

Shao, Shuai; Louisiana State University, United States

Shapter, Joe; Flinders University, Australia

Sietins, Jennifer; Army Research Laboratory, United States

Silva, Neilon; Petrobras, Brasil

Simmons, Jeff P.; US Airforce Research Laboratory, United States

Singh, Raman P.; Oklahoma State University, United States

Skwiot, Noel; Hartford Steam Boiler, United States

Smathers, David B.; HC Starck, United States

Smith, Richard; Carpenter Technology, United States

Stan, Liliana; Argonne National Laboratory, United States

Stanciu, Lia A.; Purdue University, United States

Steel, Alexander; Missile Defense Agency, United States

Stehulak, Elizabeth H.; Fort Wayne Metals, United States

Su, Yuyang; Industrial Technology Research, Taiwan

Sumardiansy, Eko; PT Indonesia Asahan Alum, Indonesia

Szczepanski, Chris; Special Metals Corp., United States

Tang, Chun; University of Nevada, Las Vegas, United States

Tang, Fei; DNV GL, United States

Thompson, Sean R.; U.S. Army Amdec, United States

Tiley, Jaimie S.; Afoser, United States
To Baben, Moritz; Factsage Family, Germany

Tonda, Richard D.; S-E-A Limited, United States

Vader, Scott J.; Vader Systems LLC; United Sates

Vader, Zachary S.; Vader Systems, LLC, United States

Valencia, Nick C.; Arrowhead Products, United States

Vos, Frans; Materials Consulting bvba, Belgium

Wais, Miachael; SLM Solutions, United States

Wakefield, Robert; Outotec, Canada

Walker, Jason; Youngstown State University, United States

Wang Dong; X'an Jiatong University, China

Wang, Peisheng; NIST, United States

Wei, Gong; Northeastern University, China

Wright, Karen; Idaho National University, United States

Wright, Ruishu; National Energy Technology, United States

Yan, Ping; China Iron \& Steel Research, China

Yashima Masatomo, Tokyo Institute of Technology, Japan

Yilong, Pu; Jiangsu Longda Group, China

Yumak, Tugrul; West Virginia University, United States

Zang, Ximin; University of Technology \& Science Liaoning, China

Zhang, Xueyuan; Gamry Instruments, United States

Zheng, Liang; Beijing Institute of Aeronautical Materials, China

Zhong, Yulin; Griffith University, Australia 